\title{
Micro-PCR Chip NTC-Thermistor Sensor-Calibration System
}

\author{
Seung-CheolLee ${ }^{1}$, Chan-Young Park ${ }^{1,2}$, Yu-Seop Kim ${ }^{1,2}$, Hye-Jeong \\ Song ${ }^{1,2}$,Jong-Dae Kim ${ }^{1,2 *}$ \\ ${ }^{1}$ Department of Convergence Software, Hallym University, Korea \\ ${ }^{2}$ Bio-IT Research Center, Hallym University, Korea \\ \{leesc4957, cypark, yskim01,hjsong, kimjd \}@hallym.ac.kr
}

\begin{abstract}
In this paper, we implement a Steinhart-Hart calibration-factor value measurement system to obtain accurate temperature measurements of a negative-temperature coefficient thermistor. The thermistor is mounted on a micro-polymerase chain reaction $(P C R)$ chip in a micro-PCR systemthat requires accurate temperature measurement and control. When obtaining the calibration-factor value, it is impossible to accurately measure and control the temperature unless the measured resistance error is set to $1 \%$. Considering this characteristic, we use the sensor of a to-be-calibrated chip to obtain accurate temperature control of a pre-calibrated chip, and we simultaneously measure the resistance value that corresponds to the temperature of the heating pattern of the precalibrated chip.After attaching a thermal pad on the heating pattern of the pre-calibrated micro-PCR chip for heat transfer, we press it as close as possible against the thermal pad on the heating pattern of the to-be-calibrated chip. When the temperature goal is reached in each of the four sections, we measure the resistance value of the to-be-calibrated chip sensor several times in order to obtain their average values. The results obtained show that the temperature error rate was within the tolerance range when the sensor of the tobe-calibrated chip measured the temperature of the heating pattern in the pre-calibrated chip. We obtained three calibration factor values from the four average resistance values. We record the calibration factor values using an electrically erasable programmable read-only memory attached on the chip, and we perform temperature calibration by again reading the calibrated values from the chip. A conventional calibration system using a constant-temperature water tank takes a long time to reach each target temperature section. However, the calibration system proposed in this paper requires a short time to reach each target temperature, and we therefore confirm that the calibration task time is less than that of conventional calibration systems.
\end{abstract}

Keywords: Polymerase Chain Reaction (PCR), Lab-on-a-chip, MEMS, Micro-PCR, NTCthermistor, Calibration

\section{Introduction}

Molecular diagnostics is a method that is often used in virus and bacteria detection, and it consists of four stages, including gene amplification and analysis using polymerase chain reactions (PCRs). Molecular diagnostics is an analytical system that is capable of genetic diagnostics in situ, which can be used instead of conventional genetic diagnostic techniques. Micro-PCR using microelectromechanical systems (MEMS), which is an example of lab-on-a-chip technology, is a technology with a fast analysis time and it requires only a small amount of reagent. It also has fast heating and cooling times, integrates various element technologies, requires low power consumption for operating devices, and has a reduced cross-contamination or biochemical risk [1]. PCR is one of the

${ }^{*}$ Corresponding Author 
most commonly used methods for generating an identical copy of certain DNA segments by amplifying a DNA sample through a heat-cycling stage at three specific temperatures. Therefore, DNA extraction from a raw sample is a required stage [2]. A micro-PCR chip obtains different types of genetic information in a short time, and facilitates genetic analysis. In addition, it is used in the diagnosis of various diseases by reacting with sample components of body fluid. Micro-PCR chips are required to have good reproducibility, sensitivity, and reliability, and to realize this, there is a need for accurate temperature measurement and control in addition to calibration for the micro-PCR chip sensor $[3,4,5,6]$. The micro-PCR chip used in this paper was fabricated using a thin printed circuit board (PCB).We heated the chamber using a heating pattern, and we measured the resistance value for the chamber temperature using a negative-temperature coefficient (NTC) thermistor at the center of the heating pattern. The measured resistance value was converted to a temperature value using the Steinhart-Hart formula. However, even if the same NTC thermistor sensor is used, there is a $1 \%$ resistance-measurement error rate. Therefore, to accurately measure the temperature of an NTC thermistor sensor, the Steinhart-Hart calibration-factor value is required, and we need a system that measures this calibration factor value. The conventionally used Steinhart-Hartcalibrationfactor value-measurement system obtained the NTC thermistor-sensor calibration-factor value of the micro-PCR chip using a constant-temperature water tank. However, the use of a constant-temperature water tank is cumbersome because it takes a long time to reach the target temperature and for temperature stabilization. In addition, we need to perform an additional task in order to use the calibration-factor value. However, by using the characteristic of the micro-PCR chip, which can increase the temperature quickly, the proposed system is an improvement on the weakness of conventional Steinhart-Hart calibration-factor value measurement system that uses a constant-temperature water tank. The proposed method is as follows. By measuring the resistance value of the temperature of a to-be-calibrated chip using the temperature control of a pre-calibrated micro-PCR chip, we can obtain the Steinhart-Hart calibration-factor value. With this method, the error-tolerance range should be considered when measuring the temperature of the precalibrated chip and that of the to-be-calibrated chip. However, when constructing the system proposed in this paper, and then experimentally measuring the error range between two chips, the error range was found to lie within the error tolerance range. Consequently, using the four averaged resistance values measured at each target temperature from the NTC thermistor sensor, three calibration-factor values could be obtained using the Armadillo library. It was then possible to record the calibration-factor values on the EEPROM memory chip mounted on the chip, read the calibration factor values again from the chip, and then calibrate the temperature. Compared to a conventional system that obtains calibration-factor values using a constant-temperature water tank, both the required space and the time needed to reach the target temperature were reduced; therefore, the overall time to perform the task has decreased. Furthermore, because the measurement error was within the tolerance range when the pre-calibrated chip's temperature was measured by a non-calibrated chip, we conclude that there is no need to calibrate the chip using a constant-temperature water tank.

In Section 2, we present the materials and methods used in our study. In Section 3, we discuss the results obtained, and we conclude the paper in Section 4.

\section{Materials and Methods}

\subsection{Device Environment}

The picture on the left-hand side of Fig. 1 shows the micro-PCR device operating chips used in the PCR experiment mentioned in this paper. The picture on the right-hand side of 
Figure 1 shows the PCR experiments that were carried out by connecting the micro-PCR chips to the device.

Figure 2 shows the micro-PCR chip used in this paper. We fabricated the micro-PCR using a thin PCB. On the opposite side of the part at which the chamber is located, the heating pattern is printed with a copper wire for the heating reagent (amplification), and at the center of the heating pattern, an NTC thermistor sensor was mounted to measure and control the temperature of the chamber. The NTC thermistor sensor is a device whose resistance changes with temperature. To measure the temperature, the resistance value is transferred to the microcontroller unit (MCU), and the MCU converts this resistance value to an analog digital converter (ADC) value. However, the NTC thermistor product mentioned in this paper has a resistance measurement error of about $1 \%$, and when this resistance value is calculated as a temperature in degrees Celsius, there is a small error when compared to the actual temperature.

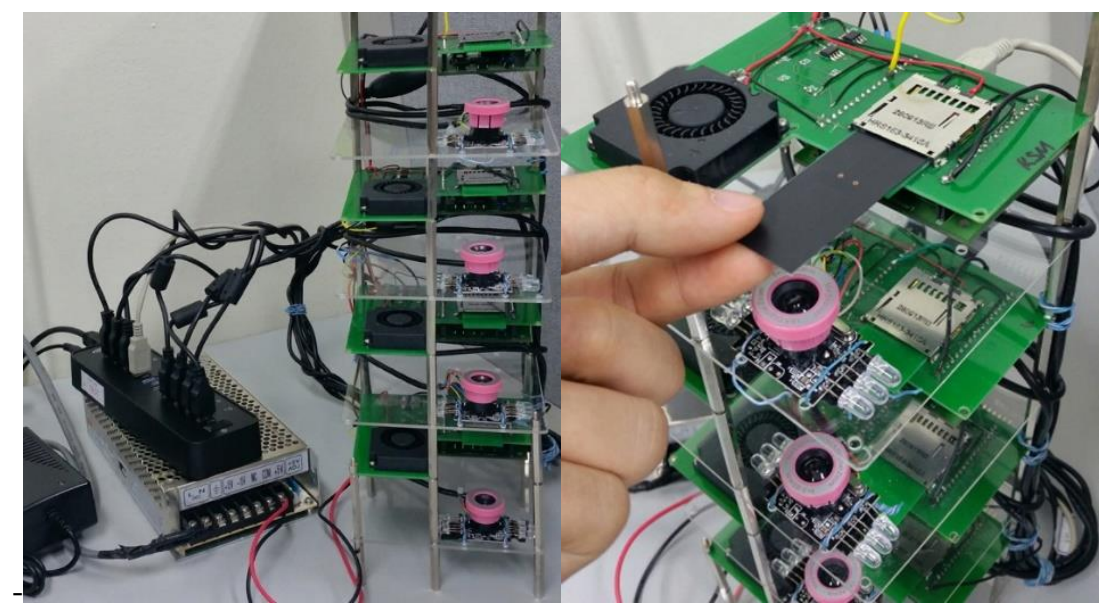

Figure 1. Micro-PCR(left) and Connecting with a Micro-PCR Chip (right)


Figure 2. Micro-PCR Chip

The conventional temperature-sensor calibration method employed by micro-PCR chips uses a constant-temperature water tank. In this method, when it reaches the four temperature values $\left(50^{\circ} \mathrm{C}, 60^{\circ} \mathrm{C}, 72^{\circ} \mathrm{C}\right.$, and $\left.95^{\circ} \mathrm{C}\right)$ required for each PCR amplification, the NTC thermistor sensor records the current resistance value. With a total of four resistance values, we obtain three calibration-factor values $(\mathrm{A}, \mathrm{B}, \mathrm{C})$ of the Steinhart-Hart formula. However, in this paper, we propose a system that can be used to calibrate a noncalibrated chip by employing a micro-PCR chip that has been calibrated in advance with 
an MCU and a constant-temperature water tank, instead of using only a constanttemperature water tank.

\subsection{Hardware Configuration}

Figure 3 shows the hardware configuration of the proposed temperature-calibration system. The MCU used in this paper was the Arduino Nano 3.1, and we used a 12V/4A $\mathrm{AC}$ adapter as the external power source that is used to apply heat to the heating pattern of the chip. In Figure 3, the "Micro PCR Chip 2" is a chip that has been calibrated in advance using a constant-temperature water tank, and it has the role of a constanttemperature water tank that applies heat indirectly to a to-be-calibrated chip, "Micro PCR Chip 1.’The heating pattern of the "Micro PCR Chip 1"was not used.

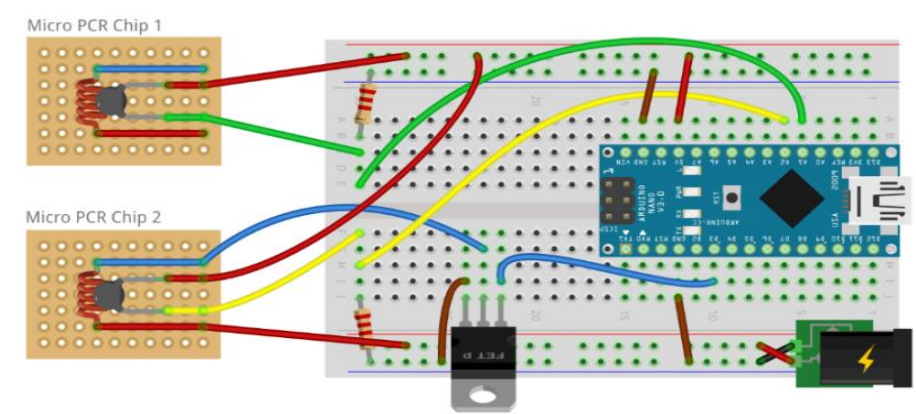

Figure 3. Calibration System Hardware Pin-out

The NTC thermistor mounted on the micro-PCR is a sensor that measures the temperature as a resistance value. In the Arduino chip, because the resistance value is obtained from the sensor as an ADC value that is within the range $0-1,023$, the ADC value is again converted to a resistance. In addition, using the Steinhart-Hart formula shown in Equation (1), we obtain the temperature in Celsius as follows.

$$
\frac{1}{T}=A+B \ln (R)+C(\ln (R))^{3}
$$

In the Steinhart-Hart formula, A, B, and C play the role of calibrating the error rate of the temperature when a resistance value with an error is converted to temperature using the Steinhart-Hart calibration-factor value. To calibrate the chip-temperature sensor, the A, $\mathrm{B}$, and $\mathrm{C}$ values of the above formula are obtained using the resistance value for each temperature of the four sections.

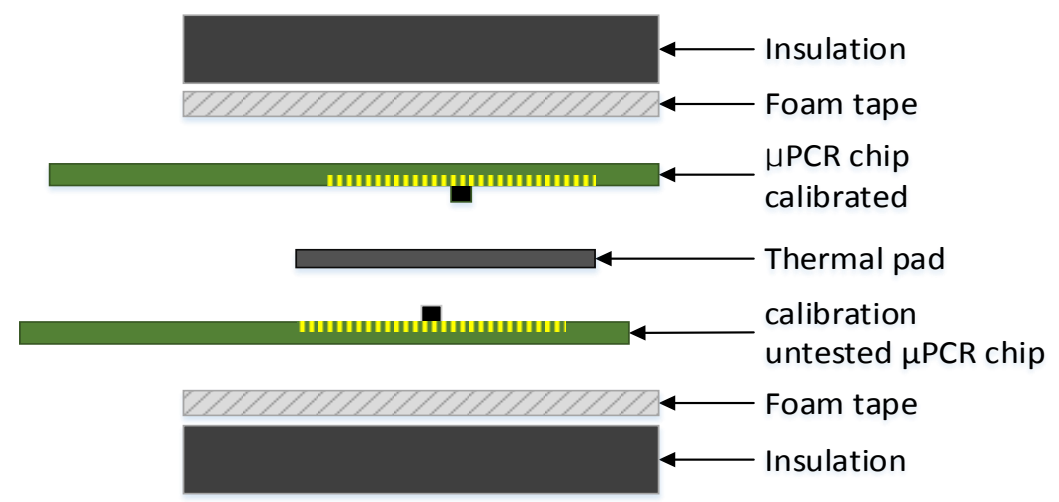

Figure 4. Configuration Diagram of the Calibration System Apparatus

Figure 4 shows the configuration of the apparatus used for the chip-calibration system. To prevent the leakage of heat from the lower and upper parts of the entire apparatus, we 
used a thermal insulator with a low thermal conductivity, and we attached a thermal pad between the micro-PCR chip and the chip to transfer heat effectively. The NTC thermistor chips located at the center of the heating pattern must not interfere with each other, and they are positioned as close as possible to each other. Then, we attached a foam tape with an empty space that is as large as the size of the heating pattern between the to-becalibrated chip and the thermal insulator to prevent the leakage of heat. Finally, by applying pressure to the top thermal insulator, all of the components are positioned to be as close as possible to each other.

\subsection{Main Firmware Modules}

The firmware of the MCU performs the following functions. When the ADC value is obtained from the NTC thermistor of the chip calibrated in advance, we obtain the temperature value using the Steinhart-Hart formula. When the target temperature is attained using the temperature value obtained from the sensor and proportional-integral derivative (PID) control, it is implemented to continuously maintain it like the function of a constant-temperature water tank. PID control is the most commonly used control method of the automatic control methods, and it enables control that maintains a constant value with combinations of three types, proportional, integral, and differential. In the proportional PID control term, we used the temperature value obtained by performing calculations using an ADC value obtained from the sensor.

\subsection{System Operation Sequence}

We performed the chip-temperature sensor-calibration task as shown in Figure 5. The micro-PCR chip to be calculated and the micro-PCR chip calibrated in advance are mounted on the hardware of the calibration system, and the apparatus is configured as shown in Figure 4. When the chip calibrated in advance reaches the four temperature sections and the temperature is maintained by PID control, the s.

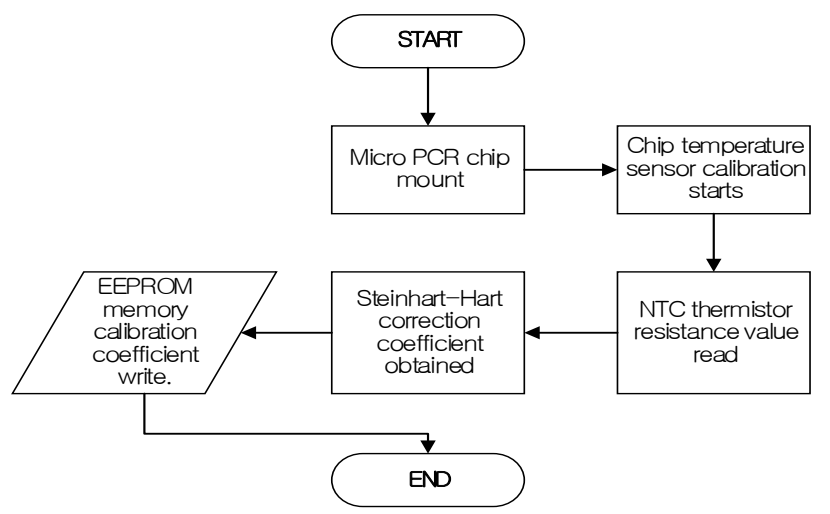

Figure 5. Flowchart of Chip-temperature Calibration System Tasks

\subsection{Experimentswith Measurement Error Range of To-be-calibrated Chip}

As shown in this paper, the pre-calibrated micro-PCR chip performs the role of a constant-temperature water tank employed in conventional chip calibration systems using a constant-temperature water tank. In conventional systems, because the temperaturecalibration task is performed by directly placing a to-be-calibrated chip in the constanttemperature water tank after waterproof treatment, there is no need to consider whether the chip sensor is properly reading the temperature. However, the system proposed in this paper does not require the direct application of heat to a to-be-calibrated chip. Moreover, instead of using an environment where a constant temperature is maintained, as in a constant-temperature water tank, heat is applied, and the temperature of the calibrated 
chip is measured using a pre-calibrated chip. This chip is placed at a specific distance outside, and there is therefore an error in the temperature value to be actually measured.

To do this, we configured the system proposed in this paper, and we measured the error range using the pre-calibrated chip instead of the to-be-calibrated chip. In other words, we used two pre-calibrated chips, and the chip that was used instead of the to-be-calibrated chip functions as the to-be-calibrated chip. However, the calibrated chip that performs the role of the to-be-calibrated chip calculates the temperature value using the calibration factor of the pertinent chip. From each chip, two temperature values are sent to a PC via a serial communication link, and data are saved every $100 \mathrm{~ms}$ for $3 \mathrm{~min}$. The data obtained for every temperature value are compiled as an Excel spreadsheet.

From the temperature values organized in Excel, we obtained the error rates by subtracting the temperature values of the two chips, and we found that when they were again converted to absolute values and the average value was obtained, the error was within the range of $0.1^{\circ} \mathrm{C}$.Two temperature values were expressed graphically, and these are shown in Figure 6. In the graph, "BOTTOM Chip" is the pre-calibrated chip and "TOP Chip" is the calibrated chip that performs the role of the to-be-calibrated chip. From the graph, we can confirm that the two temperatures were very close to each other.

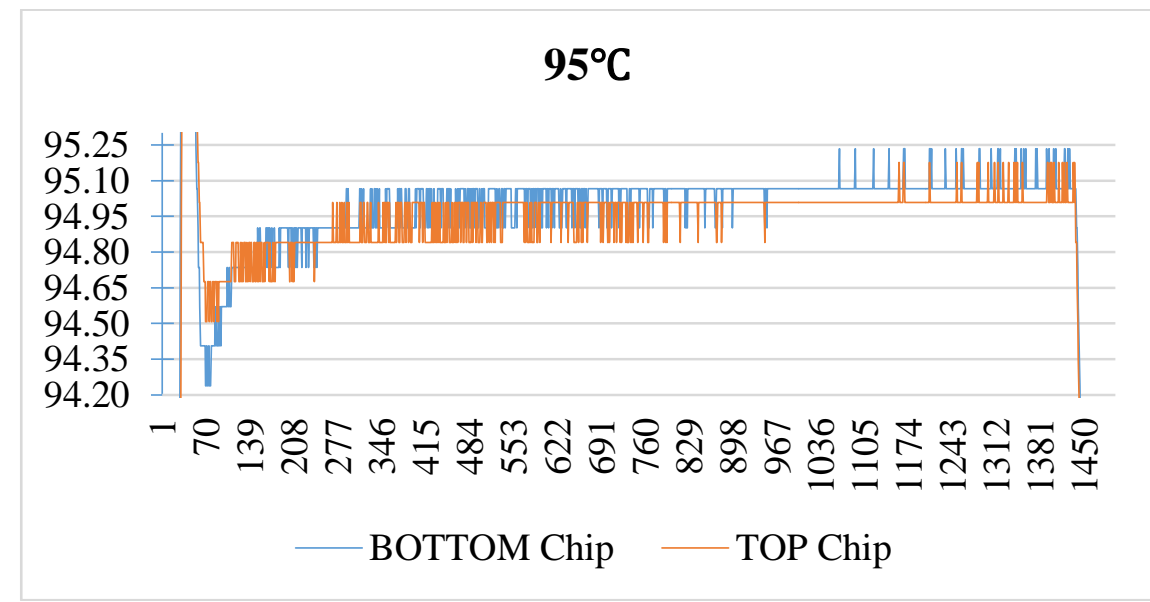

Figure 6. Graph Showing the Errors of Two Temperature Values

\section{Results}

In the experiment to measure the error range of the to-be-calibrated chip, because the error was shown to be $0.1^{\circ} \mathrm{C}$,we were able to perform the calibration task. As a result, by using the Armadillo library, we were able to obtain three Steinhart-Hart calibration-factor values from the resistance values measured at each of the four target temperatures using the NTC thermistor sensor of the uncalibrated micro-PCR chip. Therefore, we confirmed its potential to replace the chip-calibration system of a constant-temperature water tank.

\section{Conclusion}

The conventional calibration system, which requires the use of a constant-temperature water tank, significantly affects the calibration task time because a long time is required to reach each of the four target temperatures. However, for the calibration system proposed in this paper, the time that is required to reach each of the four target temperatures was measured in seconds, which is relatively short.

In our study, heat was not directly applied to theto-be-calibrated micro-PCR chip. In the experiment, where the temperature of the other chip that was heated up by itself was measured and where the error was confirmed for temperature values between two chips, the average error of the two chips was found to be $0.1{ }^{\circ} \mathrm{C}$.Because this error lies within the 
temperature error range of the PCR process, it appears to be suitable as a replacement for the conventional chip calibration system that uses a constant-temperature water tank. However, after calibrating the micro-PCR chip using the system proposed in this paper, the verification was not performed to determine whether it had been normally calibrated. This verification has to be performed using the conventional chip-calibration system with a constant-temperature water tank.Finally, by considering the result after completing the verification experiment for the PCR task, we can confirm whether the conventional calibration system can be replaced.

\section{Acknowledgments}

The research was supported by the Basic Science Research Program of the National Research Foundation of Korea (NRF), which is funded by the Ministry of Education, Science and Technology (2012R1A1A2040381), and by the Research and Business Development 2013 project of the Ministry of Trade, Industry and Energy (N0000907).

\section{References}

[1] B. H. Park, J. H. Jung, Y. T. Kim and T. S. Seo, "Development of a Fully Integrated Genetic Analysis Microsystem", (2013).

[2] C. X. Y. Yang, M. Y. Cheng and Y. J. Yang, "A DNA-extraction and polymerase-chain-reaction microchip using magnetic beads and thermo-pneumatic valves", (2010).

[3] C. Koo, M. M. Wight, H. S. Kim, O. S. Cifci and V. L. V. Diaz, "Development of a Real-Time Microchip PCR System for Portable Plant Disease Diagnosis", (2013).

[4] E. Salm, Y. S. Liu, D. Marchwiany, M. Dallas, Y. P. He, L. Razouk and A. K. Bhunia, "Erratum to: Electrical detection of dsDNA and polymerase chain reaction amplification", Biomedical Microdevices, (2011).

[5] "Fast detection of genetic information by an optimized PCR in an interchangeable chip", Biomed Microdevices, vol.14, no.1, (2012), pp.179-86.

[6] J. G. Lee, K. H. Cheong, N. Huh, S. Kim, J. W. Choi and C. K. Ko, "Microchip-based one step DNA extraction and real-time PCR in one chamber for rapid pathogen identification", Lab Chip, vol.5, (2006), pp.886-895.



\section{Authors}

Seung-Cheol Lee, he is enrolled in a master degree in Convergence Software in Hallym University. He currently studies for a master's degree in Department of Convergence Software at Hallym University. His research interests are in Bio-IT convergence, embedded system.

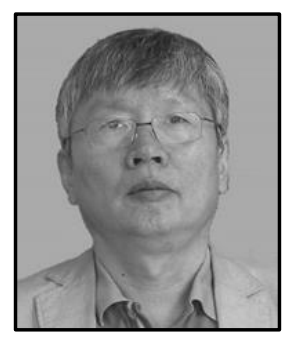

Jong-Dae Kim, he received the M.S. and the Ph.D. degrees in Electrical Engineering from Korea Advanced Institute of Science and Technology, Seoul, Korea, in 1984 and 1990, respectively. He worked for Samsung Electronics from 1988 to 2000 as an electrical engineer. He is a Professor in Department of Convergence Software, Hallym University. His recent interests focus on biomedical system and bioinformatics. 




Yu-Seop Kim, he received the Ph.D. degree in Computer Engineering from Seoul National University. He is currently a Professor in the Department of Convergence Software of Hallym University, Korea. His research interests are in the areas of bioinformatics, computational intelligence and natural language processing.



Hye-Jeong Song, she received the Ph.D. degree in Computer Engineering from Hallym University. She is a Professor in Department of Convergence Software of Hallym University, Korea. His recent interests focus on biomedical system and bioinformatics.

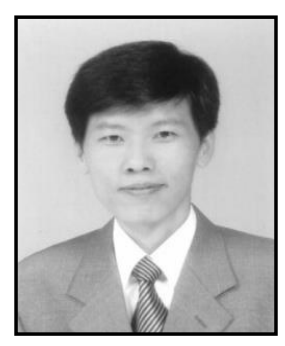

Chan-Young Park, he received the B.S. and the M.S. from Seoul National University and the Ph.D. degree from Korea Advanced Institute of Science and Technology in 1995. From 1991 to 1999, he worked at Samsung Electronics. He is currently a Professor in the Department of Convergence Software of Hallym University, Korea. His research interests are in Bio-IT convergence and sensor networks. 